\title{
Revealing the Physical Properties of GMC Complexes in the Spiral Arms of NGC 6946
}

\author{
Selçuk Topal ${ }^{1}$, Estelle Bayet ${ }^{1}$, Martin Bureau $^{1}$, Wilfred Walsh $^{2}$, and \\ Timothy A. Davis ${ }^{3}$ \\ ${ }^{1}$ Sub-department of Astrophysics, University of Oxford, Denys Wilkinson Building, Keble \\ Road, Oxford, OX1 3RH, U.K. \\ email: selcuk.topal@astro.ox.ac.uk. \\ ${ }^{2}$ Solar Energy Research Institute of Singapore (SERIS) National University of Singapore \\ (NUS), 7 Engineering Drive 1, Block E3A, 06-01, Singapore 117574 \\ ${ }^{3}$ European Southern Observatory, Karl-Schwarzschild-Str. 2, 85748, Garching bei Muenchen, \\ Germany
}

\begin{abstract}
In this study, we probe for the first time the molecular gas physical properties of several star forming regions located in the arms and inter-arms of the spiral galaxy NGC 6946. Combining our observations with additional data found in the literature, we provide in this study the most complete $\mathrm{CO}$ ladder ever obtained in these inter-arm and arm regions, i.e. the $\mathrm{CO}(1-0,2-1,3-2,4-3,6-5)$ and ${ }^{13} \mathrm{CO}(1-0,2-1)$ transitions. For each region studied, we use more precisely the large velocity gradient (LVG) assumption in order to derive the beam-averaged molecular gas physical properties. Namely, we obtained the gas kinetic temperature (i.e. 'best' $T_{\mathrm{K}}$ ), volume number gas density (i.e. 'best' $n\left(\mathrm{H}_{2}\right)$ ) and $\mathrm{CO}$ column density (i.e. 'best' $N(\mathrm{CO})$ ) which best reproduce the data for 8 regions investigated. Optical depths were also estimated for a large variety of $\mathrm{CO}$ lines in these regions. To identify the best values found, we used two complementary theoretical approaches when comparing the model predictions with the observations, i.e. the $\chi^{2}$ minimisation and the likelihood. Very different physical conditions for the molecular gas from a region to another have been obtained: $T_{\mathrm{K}}$ ranges from 10 to $250 \mathrm{~K}$, $n\left(\mathrm{H}_{2}\right)$ ranges from $10^{2.3}$ to $10^{7.0} \mathrm{~cm}^{-3}$ and $N(\mathrm{CO})$ ranges from $10^{15.0}$ to $10^{19.3} \mathrm{~cm}^{-2}$ among the arm and inter-arm regions. For each region probed, we also published for the first time the CO spectral line energy distribution (SLED) from $\mathrm{CO}(1-0)$ to $\mathrm{CO}(10-9)$ for this galaxy, mixing observations and model predictions which provide an essential insight for future follow-up observational programmes. Finally, in this work, we discuss the physical properties we obtained for each region in relation with the presence of young stellar population characteristics such as supernovae remnants (SNRs), HI holes, HII regions.
\end{abstract}

Keywords. galaxies: spiral — galaxies: ISM — ISM: molecules — ISM: clouds

\section{References}

Topal, S., Bayet, E., Bureau, M., Walsh, W., \& Davis, T. A., in prep. 